\title{
Stability analysis of solutions for fractional Langevin equation involving Hadamard-Caputo derivatives with nonlocal integral and nonperiodic boundary conditions
}

\author{
Amita Devi ${ }^{1}$, Anoop Kumar ${ }^{1}$, Thabet Abdeljawad ${ }^{2}$, and AZIZ KHAN² \\ ${ }^{1}$ Central University of Punjab \\ ${ }^{2}$ Prince Sultan University
}

November 3, 2020

\begin{abstract}
In this manuscript, we study fractional Langevin equations(FLEs) involving Hadamard-Caputo's derivative of distinct orders associated with nonlocal integral and nonperiodic boundary conditions. The Hyres-Ulam (HU) stability, existence and uniqueness(EU) of solutions are established for proposed equations. Our prospective is based on the Hadamard-Caputo's derivatives and implementation of Krasnoselskii's fixed point theorem and Banach contraction mapping principle. An application is offered to smooth the understanding of the theoretical results.
\end{abstract}

\section{Hosted file}

mma.pdf available at https://authorea.com/users/372540/articles/490537-stability-analysisof-solutions-for-fractional-langevin-equation-involving-hadamard-caputo-derivativeswith-nonlocal-integral-and-nonperiodic-boundary-conditions 